${ }^{1}$ Unidad de Enfermedades Respiratorias Hospital Regional de Talca. ¿Unidad de Paciente Crítico Clínica del Maule. Talca. ${ }^{3}$ Servicios de Radiología $y$ de ${ }^{4}$ Cirugía Hospital Regiona de Talca.

${ }^{5}$ Facultad de Ciencias de la Salud Universidad Autónoma de Chile.

Recibido el 20 de septiembre de 2012 aceptado el 8 de febrero de 2013.

Correspondencia a: Dr. Rafael Silva O. Calle 4 Norte 1652 oficina 405, Talca.

Teléfono: 71-412716 Fax: 71-209306 E-mail: rafaelsilvao@gmail. com

\section{Síndrome Hughes-Stovin. Caso clínico}

\author{
RAFAEL SILVA O. ${ }^{1,5}$, ALEJANDRO ESCOBAR ${ }^{2,5}$, \\ RODRIGO VEGA ${ }^{3}$, ROBERTO TAPIA ${ }^{4}$
}

\section{Hughes-Stovin syndrome. Report of one case}

We report a 25-year-old male admitted to a critical care unit for fever, lung opacities and acute respiratory failure. A chest angio-CAT scan showed multiple pulmonary artery aneurysms. A deep venous thrombosis of both lower limbs was also documented. A Hughes-Stovin syndrome was postulated. An inferior vena cava filter was placed. The patient received antimicrobial therapy subsiding fever and respiratory failure. Subsequently, he was treated with intravenous and oral steroids and one dose of cyclophosphamide. The patient was discharged in good conditions fifteen days after admission.

(Rev Med Chile 2013; 141: 922-926)

Key words: Behçet Syndrome; Hughes-Stovin syndrome; Vasculitis.
$\mathrm{E}$ 1 síndrome de Hughes-Stovin (SHS) es una enfermedad de baja frecuencia, descrita en $1959^{1}$. Se caracteriza por presentar aneurismas de las arterias pulmonares y trombosis venosa profunda. Esta asociación también se ha descrito en la enfermedad de Behçet (EB), sugiriendo algunas publicaciones ${ }^{2,3}$ que el SHS puede ser una forma de presentación de EB. El cuadro clínico habitualmente presenta fiebre recurrente, tos y hemoptisis ${ }^{3}$, junto con hipertensión endocraneana ${ }^{4}$.

Se presenta el caso de un varón con cefalea, fiebre, opacidades pulmonares bilaterales y múltiples aneurismas de las arterias pulmonares junto con trombosis venosa profunda de ambas extremidades inferiores.

\section{Caso clínico}

Paciente de 25 años, sin antecedentes mórbidos, con hospitalización en una clínica de Santiago de Chile en junio de 2012, por cefalea intensa, con tomografía computada cerebral y resonancia nuclear magnética cerebral normal, la que cede espontáneamente a los pocos días. Quedó sin tratamiento ni se realizaron otros estudios. No relata artritis, Raynaud, fotosensibilidad ni lesiones cutáneas. Inició el 20 de julio de 2012 cuadro febril de inicio súbito, con tos seca, dificultad respiratoria y falla respiratoria aguda con opacidades pulmonares bilaterales en radiografía de tórax. Ingresó a la unidad de paciente crítico de Clínica del Maule. Se constató relación presión arterial oxígeno/fracción inspirada oxígeno de 200. No presentaba úlceras bucales, ni genitales y fondo de ojo era normal. Inició tratamiento con imipenem $500 \mathrm{mg}$ endovenoso (EV) cada $6 \mathrm{~h}$ y vancomicina $1 \mathrm{~g}$ EV cada $12 \mathrm{~h}$ junto con hidrocortisona $100 \mathrm{mg}$ EV cada 6 h. No requirió ventilación mecánica. Entre los exámenes de laboratorio destacaba leucocitosis sin desviación a izquierda y elevación de los parámetros inflamatorios sistémicos, junto con ANCA-P Positivo. El resto de los marcadores inmunológicos eran negativos (Tabla 1). El estudio de líquido céfalo raquídeo fue normal y el panel viral respiratorio fue negativo. Hemocultivos negativos. Se realizó tomografía computada de tórax de alta resolución, que mostraba opacidades bilaterales y aumento de diámetro de los vasos pulmonares. El angio-TAC de tórax confirmó dilatación aneurismática de los vasos pulmonares en zonas basales (Figuras 1 y 2 ). Además se realizó eco Doppler de extremidades inferiores que mostró trombosis venosa profunda (TVP) en ambas extremidades. Se instaló filtro de vena cava inferior por considerar de alto riesgo iniciar tratamiento anticoagulante. A los 4 días de 
Síndrome Hughes-Stovin - R. Silva et al

Tabla 1. Exámenes de Laboratorio: inmunológicos, recuento de leucocitos, virus de la inmunodeficiencia humana (VIH) y proteína C reactiva entre el 27 de julio y el 17 de agosto de 2012

\begin{tabular}{|c|c|c|c|}
\hline & 27-7-2012 & 03-08-2012 & 17-8-2012 \\
\hline ANCA con patrón perinuclear & & Positivo & \\
\hline Anticuerpos anti ENA & & Negativo & \\
\hline Anticuerpos anti péptido citrulinado & & Negativo & \\
\hline Anticuerpos (IgG-IgM) anti cardiolipinas & & Negativo & \\
\hline Anticuerpos anti-nucleares & & Negativo & \\
\hline Anticuerpos anti DNA & & Negativo & \\
\hline Anticuerpos anti mitocondriales & & Negativo & \\
\hline Recuento leucocitos $\left(\mathrm{mm}^{3}\right)$ & 20.510 & 27.340 & 13.790 \\
\hline VIH & Negativo & & \\
\hline Proteína C reactiva (mg/L) & 256,4 & 19,6 & 7,3 \\
\hline
\end{tabular}

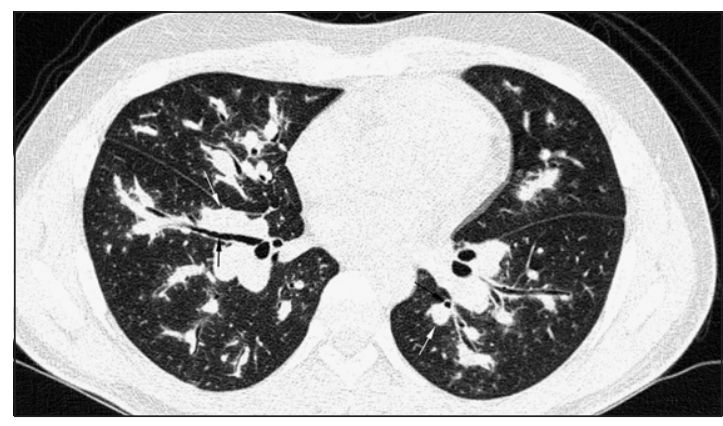

Figura 1. Tomografía computada multicorte de tórax alta resolución: innumerables dilataciones aneurismáticas fusiformes de ramas segmentarias y sub-segmentarías de la arteria pulmonar de ambos pulmones (flechas blancas). Nótese la relación de tamaño en comparación con los bronquios (flechas negras).

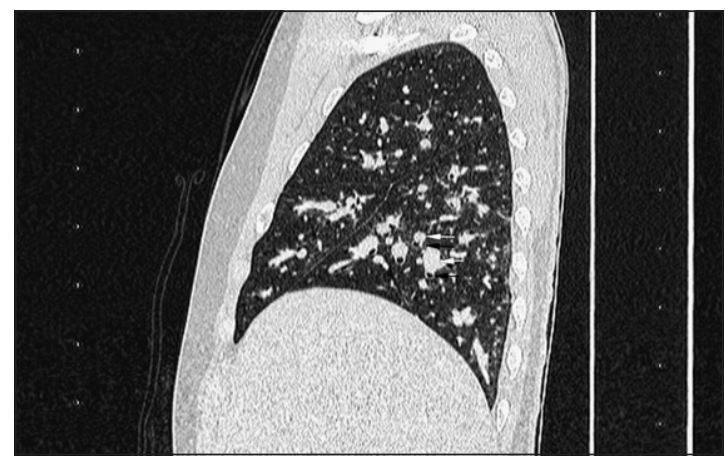

Figura 2. Reconstrucción sagital de tomografía computada multicorte de tórax: dilataciones aneurismáticas fusiformes difusas de ramas arteriales pulmonares (flecha blanca), comparando su tamaño con algunas ramas bronquiales (flecha negra). iniciado el tratamiento antibiótico cedió el cuadro febril y mejoró la falla respiratoria. Recibió 4 días de corticoides EV y luego a prednisona $1 \mathrm{mg} / \mathrm{kg}$ por vía oral ( $60 \mathrm{mg}$ día en 2 dosis). Completó 15 días de tratamiento antibiótico y al decimoquinto día de ingreso se administró ciclofosfamida 1 gramo $\mathrm{EV}$, dándose de alta en buenas condiciones.

\section{Discusión}

El síndrome de Hughes-Stovin fue descrito por 2 médicos británicos en 4 pacientes varones que presentaban aneurismas de la arteria pulmonar y TVP ${ }^{1}$.

Su etiología es desconocida y hasta el presente no hay criterios diagnósticos formalmente descritos o exámenes de laboratorio que sean específi$\cos ^{5}$. Como ya se comentó, algunos pacientes con aneurismas de arteria pulmonar y TVP pueden ser portadores de una enfermedad de Behçet y de regla esta enfermedad se debe buscar en pacientes con SHS $^{5}$.

Se han descrito menos de 40 casos de SHS en la literatura médica publicada en inglés ${ }^{5}$ y afecta preferentemente a pacientes de sexo masculino, entre los 12 y los 48 años. Ninguno de los reportes describe consanguinidad entre los casos publicados, por lo tanto, la base genética y la predisposición familiar del SHS, permanece en la nebulosa ${ }^{6-8}$.

Desde el punto de vista clínico, 25\% de los pacientes con SHS desarrollan tromboembolismo, aneurismas arteriales y oclusión vascular y, según 
esta triada, las fases clínicas serian: a) síntomas de tromboflebitis; b) formación de aneurismas pulmonares y/o bronquiales; c) rotura de aneurismas con hemoptisis masiva y muerte. Estas etapas suelen evolucionar sucesivamente. Tanto la primera como la segunda fase deben estar presentes para el diagnóstico de SHS mientras que la tercera fase es el resultado final en pacientes no tratados o no diagnosticados ${ }^{8,9}$. Por tanto, las características típicas de presentación de SHS están relacionadas con la presencia de los aneurismas pulmonares y la TVP de extremidades inferiores, tal como se presenta en nuestro paciente.

También los pacientes pueden presentar convulsiones, diplopía y cefalea, consecuencia de una hipertensión endocraneana secundaria a una trombosis de los senos venosos cerebrales ${ }^{10}$. Nuestro paciente presentó intensa cefalea intensa, que fue previa al cuadro actual, pero los estudios imagenológicos cerebrales realizados en esa oportunidad fueron normales.

Los aneurismas observados pueden ser únicos, múltiples, unilaterales o bilaterales y no sólo pueden afectar la circulación pulmonar sino que también el resto de la circulación sistémica, como hepática e ilíacas, situación que tiene menos riesgo de rotura por las bajas presiones involucradas ${ }^{11-13}$.

La tromboflebitis recurrente frecuentemente involucra grandes vasos, lo que provoca trombo embolismo, la cual se ha descrito, aparte de la habitual trombosis venosa en vena ilíaca y femoral, en vena cava y en cámaras cardiacas ${ }^{14,15}$.

La etiología y patogénesis del SHS son desconocidas, pero algunos autores proponen la vasculitis como el proceso subyacente primario ${ }^{6}$. También las embolias sépticas y los abscesos se han propuesto como causa de los aneurismas pulmonares, lo que provocaría un defecto degenerativo en las arterias bronquiales o un aneurisma micótico, como resultado de infecciones por gérmenes con virulencia de bajo grado ${ }^{1}$.

La angiodisplasia de las arterias bronquiales también se ha propuesto como causa de los cambios vasculares del SHS, alterando la nutrición adecuada y dañando los vasavasorum, afectando el tejido elástico con la consiguiente formación de aneurismas ${ }^{12}$.

Por otro lado, algunos autores han sugerido que el SHS pueda ser una manifestación parcial de la $\mathrm{EB}^{10}$, ya que esta enfermedad también puede presentar aneurismas pulmonares. En nuestro paciente no encontramos elementos que hicieran plantear el diagnóstico de EB.

La patogenia de la trombosis, tanto en SHS y en $\mathrm{EB}$, permanece no aclarada. No se han descrito en estos pacientes alteraciones trombofílicas y se ha propuesto una mutación en el gen de la protrombina con niveles aberrantes de la proteína $\mathrm{C}^{16,17}$, lo que no está demostrado.

Por último, si al SHS se agrega hiperhomocisteinemia, aumenta notablemente el riesgo de trombo embolismo venoso ${ }^{18}$.

Respecto a los hallazgos en laboratorio, no son específicos y siempre se debe realizar estudio inmunológico para el diagnóstico diferencial con enfermedades del tejido conectivo y vasculitis, especialmente poliangiitis microscópica, poliarteritis nodosa o arteritis de Takayasu. Al igual que la EB, el SHS puede tener marcadores ANCA positivos ${ }^{5}$. Además se recomienda realizar estudio de ultrasonido Doppler de extremidades inferiores para evaluar la presencia de TVP.

Respecto el estudio imagenológico, la radiografía de tórax puede mostrar opacidades lobulares o hilios pulmonares prominentes ${ }^{19}$, la angiografía pulmonar es el estándar para el diagnóstico, sin embargo, no se recomienda por el riesgo de rotura de los aneurismas pulmonares ${ }^{20}$. Por lo tanto, el examen imagenológico recomendado es el angio TAC pulmonar ${ }^{21}$ que permite obtener imágenes vasculares de alta calidad, demostrando, en estos casos, un evidente ensanchamiento del diámetro de la arteria pulmonar y dilataciones periféricas aneurismáticas de las ramas de dicha arteria. Estas situaciones se observaron en el angio TAC pulmonar de nuestro paciente (Figuras 1 y 2 ). Es posible que este procedimiento sea el método estándar de diagnóstico, por su naturaleza no invasiva, por lo fácil de realizar y por su cada vez mayor disponibilidad.

Respecto el manejo, al no existir protocolos de investigación aleatorios y controlados, no hay un estándar de tratamiento para el SHS, por lo que la terapia se ha enfocado en la línea de la $\mathrm{EB}^{10}$. Por lo tanto, se recomienda terapia inmunosupresora, combinando glucocorticoides sistémicos con ciclofosfamida. Los esteroides se administran inicialmente EV y posteriormente oral, prednisolona $1 \mathrm{mg} / \mathrm{kg} /$ día, con posterior reducción gradual. La ciclofosfamida se recomienda mensual EV en dosis ajustada por superficie corporal. Dependiendo de la respuesta clínica se puede discontinuar el 
esteroide y mantener ciclofosfamida, por lo menos, durante $1 \mathrm{año}^{22}$. Se han descrito casos, con la terapia señalada, de estabilización y hasta de regresión de los aneurismas pulmonares ${ }^{23}$.

Respecto el uso de anticoagulantes, están contraindicados por alto riesgo de hemorragia fatal, especialmente hemoptisis masiva, por rotura de los aneurismas pulmonares ${ }^{3}$.

En cuanto al pronóstico, los aneurismas de la arteria pulmonar tienen mal pronóstico, en el sentido de rotura y sangramiento ${ }^{24}$, vasculitis activa con trombosis ${ }^{24}$ y oclusión de la arteria pulmonar $^{21}$.

Finalmente, señalar que el diagnóstico precoz y el tratamiento temprano pueden ser factores que modifiquen la historia natural de esta enfer$\operatorname{medad}^{25}$, por lo cual iniciamos el tratamiento ya señalado en nuestro paciente.

\section{Conclusión}

Se presenta un paciente joven, con aneurismas múltiples en la arteria pulmonar, más una trombosis venosa bilateral de extremidades inferiores, en el cual se realiza terapia esteroidal e inmunosupresora. Es una rara enfermedad, de origen desconocido y que puede estar asociada a $\mathrm{EB}$, que actualmente no tiene guías de tratamiento validadas por protocolos.

Es el primer caso descrito en Chile y nos parece relevante comunicarlo a la comunidad médica.

\section{Referencias}

1. Hughes J, Stovin P. Segmental Pulmonary artery aneurysm with peripheral venous thrombosis. Br J Dis Chest 1959; 53: 19-27.

2. Francois MF. Is Hughes-Stovin syndrome a particular expression of Behçet's disease? Chest 1983; 83: 288.

3. Durieux P, Bletry O, Huchon G, Wechsler B, Chretien J, Godeau P. Multiple pulmonary arterial aneurysms in Behçet's disease and Hughes-Stovin syndrome. Am J Med 1981; 71: 736-41.

4. Khalid J, Weber S, Sohail S, Balaan M. Hilar Mass and papilledema on presentation. Chest 1998; 113: 228-9.

5. Khalid U, Saleem T. Hughes-Stovin Syndrome. Orphanet Journal of Rare Diseases 2011; 6: 15-26.

6. Weintraub JL, DeMayo R, Haskal ZJ, Susman J. SCVIR annual meeting filmpanel session: diagnosis and discus- sion of case 1. J Vasc Interv Radiol 2001; 12: 531-4.

7. Grembiale RD, Calderazzo M, Pelaia G, Gallelli A, Roccia F, Vero G. A case of Hughes-Stovin syndrome associated with Budd-Chiari syndrome. Caseb Repb Clin Pract Rev 2002; 3: 92-4.

8. Khalil A, Parrot A, Fartoukh M, Marsault C, Carette MF. Images incardiovascular medicine. Large pulmonary artery aneurysm rupture in Hughes-Stovin syndrome: multidetector computed tomography patternand endovascular treatment. Circulation 2006; 114: e380-1.

9. Reimold W, Emmrich J, Harmjanz D, Kochsiek K. Multiple aneurysms of the pulmonary artery following recurrent septic pulmonary embolism (Hughes-Stovin syndrome): report of 1 case. Arch Klin Med 1968; 215 : $1-18$.

10. Emad Y, Ragab Y, ShawkiAel H, Gheita T, El-Marakbi A, Salama MH. Hughes Stovin syndrome: is it incomplete Behcet's? Report of two cases and review of the literature. Clin Rheumatol 2007; 26: 1993-6.

11. Balci N, Semelka R, Noone T. Multiple pulmonary aneurysms secondary to Hughes-Stovin syndrome: demonstration by MR angiography. J Magn Reson Imaging 1998; 8: 1323-5.

12. Herb S, Hetzel M, Hetzel J, Friedrich J, Weber J. An unusual case of Hughes Stovin syndrome. Eur Respir J 1998; 11: 1191-3.

13. Veldtman GR, Dearani JA, Warnes CA. Low pressure giant pulmonary artery aneurysms in the adult: natural history and management strategies. Heart 2003; 89: 1067-70.

14. Wolpert SM, Kahn PC, Farbman K. The radiology of the Hughes-Stovin syndrome. Am J Roentgenol Radium Ther Nucl Med 1971; 112: 383-8.

15. Yagi T, Yamagishi F, Mizutani F, Sasaki Y, Sakao S, Tada Y. A case of Behçet's disease presenting with Hughes-Stovin syndrome (multiplepulmonary arterial aneurysms remitting with corticosteroid therapy). Nihon Kokyuki Gakkai Zasshi 2001; 39: 140-4.

16. Salvarani C, Calamia K, Silingardi M, Ghirarduzzi A, Olivieri I. Thrombosis associated with the prothrombin G-A20210 mutation in Behçet's disease. J Rheumatol 2000; 27: 515-6.

17. Navarro S, Ricart J, Medina P, Vayá A, Villa P, Todolí J, et al. Activated protein $\mathrm{C}$ levels in Behçet's disease and riskof venous thrombosis. Br J Haematol 2004; 126: 5506.

18. Kim H, Kim H, Park Y, Jeong Y, Kim J, Jeon D. et al. A case of Hughes-Stovin syndrome associated with hyperhomocysteinemia. Clin Rheumatol 2010; 29: 807-9.

19. Erkan F, Kiyan E, Tunaci A: Pulmonary complications of Behçet's disease. Clin Chest Med 2002; 23: 493-503. 
20. Mahlo H, Elsner K, Rieber A, Brambs H. New approach in the diagnosis of and therapy for Hughes-Stovin syndrome. AJR Am J Roentgenol 1996; 167: 817-8.

21. Ketchum E, Zamanian R, Fleischmann D. CT Angiography of Pulmonary Artery Aneurysms in Hughes-Stovin Syndrome. AJR Am J Roentgenol 2005; 185: 330-2.

22. Yazici H, Esen F. Mortality in Behçet's syndrome. Clin Exp Rheumatol 2008; 26: S138-S140

23. Lee J, Noh J, Hwang J, Kim H, Ahnn J, KohE, et al. Suc- cessful cyclophosphamide therapy with complete resolution of pulmonary artery aneurysm in Hughes-Stovin syndrome patient. Clin Rheumatol 2008; 27: 1455-8.

24. Greene R, Saleh A, Taylor A, Callaghan M, Addis B, Nzewi $\mathrm{O}$, et al. Non-invasive assessment of bleeding pulmonary artery aneurysms due to Behçet disease. Eur Radiol 1998; 8: 359-63.

25. Erkan F, Gul A, Tasali E. Pulmonary manifestations of Behcet's disease. Thorax 2001; 56: 572-8. 